\title{
H. pylori: Implicações atuais e a resistência à terapia antimicrobiana - revisão de literatura
}

\author{
Treatment and therapeutic resistance of $H$. pylori: review of literature
}

Thiago Celeira de Sousa', Ursula Tatiane de Farias Hounsell Almeida', Dauana Arruda de Oliveira Bastos ${ }^{2,3}$

Resumo Objetivo: Realizar uma revisão sobre os tratamentos e a resistência do H. pylori. Método: A pesquisa foi desenvolvida no período de Fevereiro a Junho de 2018, e realizada a partir das bases de dados: Medline, SciELO, LILACS, PubMed, e Bireme por meio dos descritores: Helicobacter Pylori, Resistência Antimicrobiana, Prevalência e Câncer Gástrico. Resultados: A busca por literatura produziu um total de 85 artigos que foram submetidos a avaliação por dois avaliadores independentes. Destes, 20 atenderam critérios de inclusão previamente estabelecidos. Conclusão: A totalidade dos estudos envolvidos nesta pesquisa nos mostram uma elevada prevalência da infecção por H. pylori e alta taxa de resistência para muitos antibióticos, tendo isto um impacto preocupante em um continente com áreas de alta prevalência de contaminação e também com altas taxas de incidência de doenças graves relacionadas a infecção, como lesões pré-neoplásicas e o adenocarcinoma gástrico. As taxas de resistência á claritomicina variaram entre $11,1 \%$ a $47 \%$ nos resultados dos artigos selecionados para este estudo, sendo portando o antimicrobiano que apresentou a maior taxa de resistência. Em relação ao tratamento, os 3 principais guidelines utilizados (Colégio Americano, Consenso Brasileiro e Consenso Europeu) concordam em relação á primeira linha de tratamento, porém os consensos Europeu e Americano propõem o uso de metronidazol como alternativa á amoxicilina nesta linha de tratamento.

Descritores: Helicobacter Pylori; resistência microbiana a medicamentos; câncer gástrico; tratamento farmacológico.

Summary Purpose: Reviewer of the treatments and resistance of H. pylori. Method: The research was carried from February to June 2018, and performed from the databases: Medline, SciELO, LILACS, PubMed, and Bireme by means of the descriptors: Helicobacter Pylori, Antimicrobial Resistance, Prevalence and Gastric Cancer. Results: The search for literature produced a total of 85 articles that were submitted to evaluation by two independent evaluators. Of these, 20 met previously established inclusion criteria. Conclusion: All the studies involved in this study show a high prevalence of $H$. pylori infection and a high resistance rate for many antibiotics. This has a worrying impact in a continent with areas of high contamination prevalence and also with high rates of incidence of serious infection-related diseases such as pre-neoplastic lesions and gastric adenocarcinoma. The rates of resistance to clarithromycin ranged from $11.1 \%$ to $47 \%$ in the results of the articles selected for this study, with the antimicrobial having the highest resistance rate. Regarding treatment, the 3 main guidelines used (American College, Brazilian Consensus and European Consensus) agree on the first line of treatment, but the European and American consensus proposes the use of metronidazole as an alternative to amoxicillin in this treatment line.

Keywords: Helicobacter pylori; antimicrobial resistance; gastric cancer; drug therapy.

${ }^{1}$ Faculdade Metropolitana da Amazônia - FAMAZ, Belém, PA, Brasil

${ }^{2}$ Universidade Federal de São Paulo - UNIFESP, Escola Paulista de Medicina - EMP, São Paulo, SP, Brasil

${ }^{3}$ Hospital Ophir Loyola, Belém, PA, Brasil

Fonte de financiamento: nenhuma

Conflito de interesses: Os autores declaram não haver conflitos de interesse.

Recebido: Julho 03, 2018

Aceito: Julho 15, 2018

Trabalho realizado na Faculdade Metropolitana da Amazônia - FAMAZ, Belém, PA, Brasil.

Copyright Sousa et al. Este é um artigo publicado em acesso aberto (Open Access) sob a licença Creative Commons Attribution, que permite uso, distribuição e reprodução em qualquer meio, sem restrições desde que o trabalho original seja corretamente citado. 


\section{Introdução}

A infecção pelo Helicobacter pylori (H. pylori) é a patologia mais frequente em todo o mundo, com prevalência média de $50 \%$, sendo o humano seu principal reservatório ${ }^{1}$. É uma bactéria espiral, gram-negativa que está ligada à patogênese de diversas afecções do sistema digestivo. A infecção causada pelo H. pylori na mucosa gástrica está comumente associada com processos inflamatórios, resultando em gastrites, doença ulcerosa péptica e processos neoplásicos, tais como o adenorcarcinoma e o linfoma MALT (Mucosa Associated Lymphoid Tissue $)^{1-4}$.

A infeção por H. pylori é provavelmente a mais frequente em todo o mundo, no entanto sua prevalência vem reduzindo em algumas regiões. Tipicamente a infeção é adquirida durante a infância, dentro do núcleo familiar. Estudos demonstram que há diferença entre a população acometida dos países em desenvolvimento e nos desenvolvidos, sendo nos primeiros uma porcentagem maior que no segundo. A taxa de infeção nos países industrializados diminuiu substancialmente nas últimas décadas, provavelmente devido à melhoria das condições de higiene ${ }^{2,4,5}$. Já em relação à realidade nacional, um estudo recente envolvendo amostras de 45.206 biópsias gástricas em um laboratório brasileiro de anatomia patológica, revelou uma prevalência de $30,93 \%$ de amostras positivas para H. Pylori $^{6}$.

Esta elevada prevalência vem preocupando os pesquisadores, pois sabe-se que pelo menos $90 \%$ dos tumores estão relacionados com a infecção por H. pylori ${ }^{7}$, levando a Organização Mundial da Saúde (OMS) a reconhecer o patógeno como carcinogênico para o câncer gástrico, segundo a classificação de IARC - International Agency for Research on Cancer, no grupo I (carcinogênico para humanos) $)^{1,8}$.

No manejo do H. pylori, a resistência antimicrobiana é considerada o principal fator relacionado á falha na erradicação do patógeno ${ }^{4,7}$. A suscetibilidade do H. pylori para antibióticos pode ser ligeiramente variável em particulares áreas geográficas de um mesmo país, assim como de diferentes países, sendo diretamente influenciada pelo uso prévio destes medicamentos. Porém, mesmo que haja sucesso de um esquema terapêutico em uma determinada comunidade, tais resultados não podem ser generalizados. $\mathrm{O}$ mais indicado seria a adoção de uma terapêutica baseada em conhecimentos prévios acerca da resistência microbiana em uma comunidade local, o que tem sido difícil na maioria dos países em desenvolvimento?.

O tratamento primário de acordo com o $4^{\circ}$ Consenso Brasileiro de $H$. pylori para a infecção por este microrganismo consiste no uso de um inibidor de bomba de prótons (IBP) associado a amoxicilina e claritromicina ou da quadrupla terapia com uso de IBP, sal de bismuto, tetraciclina e metronidazol. Entretanto, vários outros antibióticos vêm sido sugeridos para uso em combinação com os IBP's, incluindo furazolidona e eritromicina ${ }^{1,5,7}$.

Perante a falha no tratamento inicial, várias recomendações terapêuticas têm sido propostas. Dentre elas está o uso da triplo terapia substituindo a claritromicina por levofloxacino ou quádrupla terapia conforme já descrito acima por um período de 10 a 14 dias,

Diante dos dados expostos acima, e com a crescente preocupação em relação ao crescimento da resistência antimicrobiana, o presente artigo trata-se de uma revisão sistemática da literatura com tema: tratamento e resistência antimicrobiana do H. pylori, visando à condensação e a sistematização dos atuais conhecimentos, dados e estatísticas relacionados ao tema.

\section{Método}

Para a busca dos artigos foi feito um levantamento nas bases de dados eletrônicas Medline, Pubmed, Lilacs, Scielo e Bireme, datados de 2008 até 2018, escritos em português, espanhol ou inglês. Para a pesquisa, palavras-chaves foram incluídas, tais como: Helicobacter pylori, resistência, tratamento, epidemiologia, câncer gástrico. Não houve restrição em termos de idade e as patologias básicas dos participantes.

Foram incluídos artigos que apresentaram a prevalência da resistência do $H$. pylori a diversos antibióticos e / ou que relataram mutações relacionadas à resistência; que apresentaram características inerentes ao patógeno, tais como epidemiologia, fisiopatologia, diagnóstico e relação com câncer gástrico, e também que apresentaram guias ou opções de tratamento para a erradicação do patógeno. Foram excluídos artigos não disponíveis na íntegra ou para download gratuito, e artigos que não atenderam aos objetivos desta pesquisa ou que descreviam apenas superficialmente o tema tratado.

Após a seleção dos artigos, os dados foram extraídos em um formato padronizado. De cada um dos artigos selecionados foram obtidos dados sobre particularidades do microrganismo em estudo, tais como fisiopatologia, epidemiologia, diagnóstico, tratamento e relação com câncer gástrico; sensibilidade e resistência 
aos esquemas antimicrobianos disponíveis, tipo de mutações encontradas, tipo de testes microbiológicos realizados para avaliar a sensibilidade das amostras e o número de participantes do estudo.

\section{Resultados}

A busca por literatura produziu um total de 85 artigos compreendidos entre os anos de 2013 e 2018, das bases de dados Medline, Lilacs, Pubmed, Bireme e Scielo, que foram submetidos a avaliação por dois avaliadores independentes. Destes, 20 atenderam aos critérios de inclusão previamente estabelecidos. Foram excluídos os artigos em que a metodologia utilizada não foi adequada e em que a resistência antimicrobiana não foi claramente descrita. Dentre os artigos escolhidos, 1 datava do ano de 2008, 1 de 2013, 5 de 2016, 1 de 2017 e 12 de 2018.

Em relação à qualidade metodológica dos estudos avaliados foram enquadrados como "qualidade adequada" na maioria dos aspectos avaliados; todos os artigos descreviam claramente a metodologia utilizada. Em relação ao idioma dos artigos selecionados, 18 eram em língua inglesa e 2 em língua portuguesa. Dentre estes artigos, 8 relataram a problemática da resistência antimicrobiana, 8 trataram sobre os aspectos gerais do microrganismo (epidemiologia, fisiopatologia, diagnóstico e relação com câncer gástrico), e 4 artigos descreveram aspectos do tratamento e os novos guidelines para o mesmo e todos os estudos relataram as conclusões feita pelos autores.

\section{Discussão}

\section{$O$ problema da resistência}

Verificou-se que a base molecular, no H. Pylori, de resistência aos danos, resulta do mecanismo de efluxo do fármaco ou pode ser atribuída à presença de mutações. O efluxo de drogas resulta da ação de uma proteína transportadora que pode resultar de uma redução da concentração antimicrobiana dentro de uma célula bacteriana, aumentando suas chances de sobrevivência na presença de antimicrobianos. No entanto, o principal mecanismo de resistência do germe resulta da aquisição de mutações pontuais ${ }^{10}$.

Como já citado anteriormente, existem várias implicações para a diminuição da eficácia da tripla terapia padrão, sendo a mais importante o aumento da resistência do $H$. pylori à claritromicina ${ }^{11}$. A taxa de resistência global à claritromicina na Europa aumentou de $9 \%$ em 1998 para 17,5\% em 2008-9. Este aumento verificou-se na maior parte da Europa, atingindo atualmente uma prevalência maior que 20\%, considerada uma taxa de resistência elevada na maioria dos países da Europa central, ocidental e do sul da Europa ${ }^{11}$. Este resultado complementa as informações obtidas com um estudo realizado no Brasil envolvendo diferentes regiões para a determinação molecular da resistência genotípica primária de H. pylori à claritomicina e fluorquinolonas, que detectou resistência à claritromicina e a fluoroquinolona em pacientes ${ }^{12}$.

Estas taxas de resistência vêm apresentando concordância com outros estudos nacionais, incluindo um estudo envolvendo 342 pacientes no Hospital de Clínicas de Porto Alegre, no sul do Brasil, nos quais se demonstrou resistência a claritromicina $(11,1 \%)$, amoxicilina $(1,9 \%)$ e a ambos medicamentos utilizados em conjunto $^{13}$. Outro estudo, realizado em uma grande cidade no Sudeste do Brasil, observou uma taxa de resistência à claritromicina $(12,5 \%)$ e a fluoroquinolonas $(11,1 \%)$, sendo incomun a resistencia dupla a esses antibióticos ${ }^{10,13,14}$.

Já em relação ao cenário mundial, em um estudo realizado no continente africano, com amostras isoladas apresentou resistência do H. pylori nas seguintes variações: metronidazol $(74,1-, 77,4 \%)$, amoxicilina $(68,6-76,6 \%)$, tetraciclina $(26,7-31,8 \%)$, claritromicina $(44,552,9 \%)$, e quinolonas $(12,8-21,9 \%)$, mostrando assim taxas muito elevadas em comparação aos estudos citados anteriormente ${ }^{13}$. Outra pesquisa, realizada na Palestina para sensibilidade do H. pylori a seis antibióticos, a ciprofloxacina foi a droga mais eficaz contra o patógeno ( $0 \%$ de resistência), seguida pela levofloxacina $(0 \%)$, moxifloxacina ( $3 \%)$ e amoxicilina ( $18 \%)$. O metronidazol e a claritromicina foram os menos eficazes, com taxas de resistência de $100 \%$ e $47 \%$, respectivamente ${ }^{10,12}$.

Sendo assim, tendo conhecimento prévio sobre a alta prevalência de resistência antimicrobiana relacionada ao H. Pylori, como os estudos vêm demonstrando, compreende-se que, para qualquer regime, a taxa de erradicação pode ser prevista se as taxas de cura forem conhecidas por cepas suscetíveis e resistentes e a prevalência de resistência na população. Para um mesmo paciente, uma história de uso prévio de qualquer um dos principais antibióticos propostos, identificará a provável resistência, mesmo com as baixas taxas de resistência na população ${ }^{13}$. 


\section{Relação com câncer gástrico}

Como consequência da estreita associação da infecção por H. pylori e câncer gástrico, a Agência Internacional de Pesquisa sobre Câncer (IARC) classificou este patógeno como carcinogênico classe I (carcinogênico para humanos) em 2012, baseando-se em evidências epidemiológicas sobre seu papel na patogênese dos adenocarcinomas gástricos ${ }^{3,4}$.

Uma combinação da alta prevalência de infecção por H. pylori e subsequente risco elevado para a associação com câncer gástrico resulta em um alto risco atribuível para o desenvolvimento desta afecção. Com base nos 952.000 novos casos de câncer gástricos diagnosticados anualmente em todo o mundo, foi recentemente estimado que 753.000 (79\%) destes poderia, ser atribuídos à infecção por $H$. pylori, com outros 13.000 linfomas gástricos também associados a este patógeno¹.

A erradicação do H. pylori reduz o risco de desenvolvimento de lesões precursoras do câncer gástrico9 . Tal atitude, prevenindo a progressão da carcinogênese gástrica, pode ser vista como uma estratégia de prevenção primária na população geral sintomática com esta infeção, sendo indicado, pelo IV Consenso Brasileiro em H. pylori, o tratamento de erradicação deste patógeno aos pacientes com dispepsia e com a presença de desta bactéria confirmada ${ }^{7}$. Contudo, a elevada prevalência da infeção, os custos e os efeitos adversos da terapêutica de erradicação suscitam dúvidas quanto ao custo-efetividade desta estratégia ${ }^{3,7}$.

Sendo assim, quando a gastrite crônica ativa é revertida pela erradicação do $H$. Pylori, a cascata carcinogênica é interrompida. O momento ideal para a erradicação do H. Pylori, no câncer gástrico, é anterior ao surgimento de condições pré-neoplásicas (gastrite atrófica e metaplasia intestinal). Além disso, mesmo com a condição pré-neoplásica já estabelecida, a erradicação do $H$. pylori reduz o risco de câncer gástrico ${ }^{7}$.

\section{Novas implicações no tratamento para infecção pelo Helicobacter pylori}

Durante as últimas duas décadas a tripla terapia com IBP, amoxicilina, e claritromicina por sete dias era largamente utilizada. No entanto várias pesquisas vêm demonstrando falhas neste esquema em decorrência da resistência microbiana a claritromicina e ao tempo de uso, além de buscarem melhores escolhas terapêuticas ${ }^{7,9,15}$.

Segundo o IV Consenso Brasileiro em Infecção por H. pylori (2018), a triplo terapia padrão, que consiste numa combinação de IBP, amoxicilina, e claritromicina por 14 dias é recomendada como o tratamento de primeira linha, a fim de alcançar altas taxas de erradicação do patógeno ${ }^{7,16}$. Já o Colégio Americano de Gastroenterologia, assim como o Maastricht V/Florence Consensus (Consenso Europeu), inclui o metronidazol como opção à amoxicilina no tratamento de $1^{\mathrm{a}}$ linha, sendo indicada em regiões onde sabe-se que a resistência a claritromicina é menor que $15 \%$ e em doentes sem história prévia de exposição a macrolídeos por qualquer motivo $^{13}$. O Consenso Europeu ainda acrescenta que a duração da terapia pode ser diminuída para 10 dias, caso seja comprovadamente eficaz localmente ${ }^{13}$ (Tabela 1).

Como alternativa à primeira escolha padrão, principalmente em regiões que são comprovadas resistência a claritromicina, quádrupla terapia com IBP, sais de bismuto, tetraciclina e metronidazol de 10 a 14 dias ou a terapia concomitante com IBP, amoxicilina, claritromicina e metronidazol por 14 dias $^{7}$. Tanto o Colégio Americano, quanto o Consenso Europeu, também ultilizam este esquema como opção alternativa a terapia ${ }^{13}$ (Tabela 1).

O uso de metronidazol pode ser problemático por conta dos altos níveis de resistência do H. pylori a este medicamento. A eficácia do tratamento é determinada pelas baixas taxas de resistência dupla do patógeno à claritromicina e aos nitroimidazóis, e se aceita que a porcentagem de cura possa atingir aproximadamente $90 \%$ nos casos em que a resistência dupla não exceda $15 \%$.

Um estudo recente afirma que o uso de sais de bismuto aumenta a taxa de cura se comparado ao não uso do mesmo $^{10}$, entretanto, outra pesquisa afirma que o não uso do bismuto é eficaz e seguro como primeira linha de tratamento de erradicação em populações com altas taxas de resistência a antibióticos ${ }^{17}$. Mesmo existindo alternativas ao tratamento de primeira linha, a terapia mais indicada por muitos autores ainda é a que utiliza três medicamentos8,9,15,16,18.

Para o manejo de pacientes com alergia à penicilina, a combinação preconizada pelo Consenso Brasileiro consiste na associação de IBP, claritromicina e levofloxacina por 14 dias; IBP, associado à doxiclina por 14 dias ou IBP associado à tetraciclina, metronidazol e bismuto por 14 dias $^{7}$ (Tabela 1). Este último regime também 
é adotado pelo Consenso Americano ${ }^{13}$, porém o consenso Europeu adota outra linha de terapia para estes casos específicos de alergia à penincilina, como uma associação entre IBP, metronidazol e tetraciclina por 10 dias $^{13}$.

Existem várias explicações para a diminuição da eficácia da tripla terapia como a não adesão ao tratamento, alta acidez gástrica, alta carga bacteriana e virulência das cepas bacterianas, mas o mais importante é o aumento da resistência do H. pylori à claritromicina ${ }^{18}$. Apesar deste fato, um estudo brasileiro realizou teste molecular para avaliar a resistência a claritromicina associada ao metronidazol e sugere que ainda sejam usadas essas medicações tanto para primeira como para segunda escolha de tratamento ${ }^{18}$.

Diante do fato de resistência microbiana, a recomendação para uso na segunda linha de tratamento consiste na triplo terapia com levofloxacino em substituição a claritromicina ou na quádrupla terapia com IBP, sais de bismuto, tetraciclina e metronidazol, ambos por 10 a 14 dias $^{7,9}$. Caso seja escolhida a primeira e a mesma falhe, é aconselhado usar a quádrupla terapia como terceira linha de tratamento e vice versa ${ }^{7,9}$ (Tabela 2). Segundo um estudo de meta análise, comparando esses dois tratamentos, concluiu que o uso da triplo terapia com levofloxacino é mais eficaz que a quádrupla terapia por 10 dias ${ }^{19}$. No entanto, em outro estudo de meta análise realizado demonstrou diferença a favor do tratamento de segunda linha por 14 dias, independente de se usar tripla ou quádrupla terapia com bismuto ${ }^{16}$.

O Colégio Americano e o Consenso Europeu também adotam a triplo terapia com levofloxacina como tratamento de segunda linha, porém incluem na combinação da terapia quádrupla de bismuto a rifabutina, como uma opção em casos de alta resistência às quinolonas ${ }^{13,17}$. Regimes quádruplos contendo bismuto e empregando furazolidona no lugar do metronidazol mostraram bons resultados no Brasil ${ }^{8}$. No entanto, a suspensão da comercialização de furazolidona e a recente indisponibilidade de sais de bismuto no Brasil tem sido limitantes no uso desta opção terapêutica ${ }^{7}$.

Ao redor do mundo o regime de erradicação para H. Pylori mais usado é a triplo terapia com IBP, amoxicilina e levofloxacino após a falha na primeira linha de tratamento com claritromicina ${ }^{20}$. Após três falhas terapêuticas, o tratamento deve-se restringir a casos especiais e devem ser usados testes genotipicos de suscetibilidade antimicrobiana para guiar o mesmo $^{7,9}$.

Tabela 1. Drogas e duração recomendada para primeira linha de terapia

\begin{tabular}{llc}
\hline \multicolumn{1}{c}{ Recomendação $\mathbf{1}^{\text {a }}$ Linha terapia } & \multicolumn{1}{c}{ Medicamento } & Tempo de tratamento \\
$\begin{array}{l}\text { Triplo terapia Padrão } \\
\left(4^{\circ} \text { consenso brasileiro }\right)\end{array}$ & IBP, amoxicilina, claritromicina & 14 dias \\
\hline EUA e Europa & IBP, metronidazol, claritromicina & $10-14$ dias \\
\hline Quádrupla terapia com bismuto & IBP, sais de bismuto, tetraciclina e metronidazol & $10-14$ dias \\
\hline Quádrupla terapia sem bismuto & IBP, amoxicilina, claritromicina e metronidazol & 14 dias \\
\hline Alérgicos a penicilina & IBP, claritromicina e levofloxacina & 14 dias \\
\hline
\end{tabular}

Fonte: Coelho et al. ${ }^{7}$ e Malfertheiner et al. ${ }^{13}$.

Tabela 2. Drogas e duração recomendada para segunda e terceira linha de terapia nos casos de falha da terapia primária padrão

\begin{tabular}{llc}
\hline \multicolumn{1}{c}{ Recomendação $\mathbf{2}^{\text {a }}$ Linha terapia } & Medicamento & Tempo de tratamento \\
\hline Tripla terapia com levofloxacino & IBP, amoxicilina, levofloxacino & $10-14$ dias \\
\hline Quadrupla terapia com bismuto & IBP, sais de bismuto, tetraciclina e metronidazol & $10-14$ dias \\
\hline
\end{tabular}

Fonte: Coelho et al. ${ }^{7}$ e Zagari et al. ${ }^{9}$. 


\section{Conclusão}

A resistência antibiótica do H. pylori é um problema crescente em todo o mundo e é a principal causa de falhas na erradicação com os esquemas atuais. Os artigos incluídos neste estudo demonstraram taxas de resistências preocupantes à claritromicina, variando entre $11,1 \%$ á $47 \%$ e as quinolonas de $21,9 \%$. Para reverter tal situação novas estratégias estão sendo sugeridas, entre elas o aumento do tempo de tratamento para 14 dias, esquema quádruplo de medicamentos, inclusão de sais de bismuto e o uso de novos antibióticos como as fluoroquinolonas. Mesmo diante de várias possibilidades de tratamento, a triplo terapia padrão ainda é a mais utilizada e recomendada como primeira escolha. E que na falha deste esquema, após três tentativas de retratamento, deve-se prosseguir com a investigação genotípica sobre a sensibilidade ao antimicrobiano para que assim, possa-se obter o sucesso da terapia.

\section{Referências}

1. Park JY, Forman D, Waskito LA, Yamaoka Y, Crabtree JE. Epidemiology of Helicobacter pylori and CagA-Positive infections and global variations in gastric cancer. Toxins (Basel). 2018;10(4):163. http://dx.doi.org/10.3390/toxins10040163. PMid:29671784.

2. Passos MCF. Drug resistance in Helicobacter pylori. Arq Gastroenterol. 2016;53(4):213-4. http://dx.doi.org/10.1590/S0004-28032016000400001. PMid:27706448.

3. Teixeira TF, Souza IKF, Rocha RDR. Helicobacter pylori: Infecção, diagnóstico laboratorial e tratamento. Percurso Acadêmico. 2016;6(12):481504. http://dx.doi.org/10.5752/P.2236-0603.2016v6n12p481.

4. Crabtree JE, Wessler S. Special Issue "H. pylori Virulence Factors in the Induction of Gastric Cancer". Toxins (Basel). 2018;10(5):176. http:// dx.doi.org/10.3390/toxins10050176. PMid:29701631.

5. Vesga FJ, Moreno Y, Ferrús MA, Campos C, Trespalacios AA. Detection of Helicobacter pylori in drinking water treatment plants in Bogotá, Colombia, using cultural and molecular techniques. Int J Hyg Environ Health. 2018;221(4):595-601. http://dx.doi.org/10.1016/j. ijheh.2018.04.010. PMid:29709385.

6. Zamani M, Ebrahimtabar F, Zamani V, Miller WH, Alizadeh-Navaei R, Shokri-Shirvani J, et al. Systematic review with meta-analysis: the worldwide prevalence of Helicobacter pylori infection. Aliment Pharmacol Ther. 2018;47(7):868-76. http://dx.doi.org/10.1111/apt.14561. PMid:29430669.

7. Coelho LG, Maguinilk I, Zaterka S, Parente JM, Passos MCF, Moraes-Filho JPP, et al. IV th Brazilian Consensus Conference on Helicobacter pylori infection. Arq Gastroenterol. 2018;55(2):97-121. http://dx.doi.org/10.1590/s0004-2803.201800000-20.

8. Luscenti RS, Gatti LL. Diagnóstico molecular da infecção pelo Helicobacter Pylori em mucosa gástrica. Rev Para Med. 2008;22(1):21-26.

9. Zagari RM, Rabitti S, Eusebi LH, Bazzoli F. Treatment of Helicobacter pylori infection: a clinical practice update. Eur J Clin Invest. 2018;48(1):12857. http://dx.doi.org/10.1111/eci.12857. PMid:29144559.

10. Long X, Chen Q, Yu L, Liang X, Liu W, Lu H. Bismuth improves efficacy of proton-pump inhibitor clarithromycin, metronidazole triple Helicobacter pylori therapy despite a high prevalence of antimicrobial resistance. China. Helicobacter. 2018;23(3):e12485. http://dx.doi. org/10.1111/hel.12485. PMid:29696736.

11. Abdoh Q, Kharraz L, Ayoub K, Khraim J, Awad W, Sbeah A, et al. Helicobacter pylori resistance to antibiotics at the An-Najah National University Hospital: a cross-sectional study. The Lancet. 2018;391(S32):32. http://dx.doi.org/10.1016/S0140-6736(18)30398-2.

12. Sanches BS, Martins GM, Lima K, Cota B, Moretzsohn LD, Ribeiro LT, et al. Detection of Helicobacter pylori resistance to clarithromycin and fluoroquinolones in Brazil: a national survey. World J Gastroenterol. 2016;22(33):7587-94. http://dx.doi.org/10.3748/wjg.v22.i33.7587. PMid:27672279.

13. Malfertheiner P, Megraud F, O'Morain CA, Gisbert JP, Kuipers EJ, Axon AT, et al. Management of Helicobacter pylori infection-the Maastricht V/Florence Consensus Report. Gut. 2016;66(1):6-30. PMid:27707777.

14. Crabtree JE, Wessler S. Special Issue “H. pylori Virulence Factors in the Induction of Gastric Cancer". Toxins (Basel). 2018;10(5):176. http:// dx.doi.org/10.3390/toxins10050176. PMid:29701631.

15. Gatta L, Scarpignato C, Fiorini G, Belsey J, Saracino IM, Ricci C, et al. Impact of primary antibiotic resistance on the effectiveness of sequential therapy for Helicobacter pylori infection: lessons from a 5-year study on a large number of strains. Aliment Pharmacol Ther. 2018;47(9):1261-9. http://dx.doi.org/10.1111/apt.14597. PMid:29536561.

16. Muñoz N, Sánchez-Delgado J, Baylina M, Puig I, López-Góngora S, Suarez D, et al. Systematic review, meta-analysis, and meta-regression: Successful second-line treatment for Helicobacter pylori. Helicobacter. 2018;23(3):e12488. http://dx.doi.org/10.1111/hel.12488. PMid:29663581.

17. Georgopoulos SD, Papastergiou V, Martinez-Gonzalez B, Xirouchakis E, Familias I, Sgouras D, et al. Hybrid therapy as first-line regimen for Helicobacter pylori eradication in a high clarithromycin resistance area: a prospective open-label trial. Ann Gastroenterol. 2018;31(2):205-10. PMid:29507467. 
18. Martins GM, Sanches BSF, Moretzsohn LD, Lima KS, Cota BDCV, Coelho LGV. Molecular detection of clarithromycin and fluoroquinolones resistance in Helicobacter pylori infection, directly applied to gastric biopsies, in an urban Brazilian population. Arq Gastroenterol. 2016;53(2):113-7. http://dx.doi.org/10.1590/S0004-28032016000200012. PMid:27305419.

19. Marin AC, McNicholl AG, Gisbert JP. A review of rescue regimens after clarithromycincontaining triple therapy failure (for Helicobacter pylori eradication). Expert Opin Pharmacother. 2013;14(7):843-61. http://dx.doi.org/10.1517/14656566.2013.782286. PMid:23537368.

20. Chey WD, Leontiadis GI, Howden CW, Moss SF. ACG clinical guideline: treatment of Helicobacter pylori infection. Am J Gastroenterol. 2017;112(2):212-39. http://dx.doi.org/10.1038/ajg.2016.563. PMid:28071659.

\section{Autor correspondente}

Thiago Celeira de Sousa

Faculdade Metropolitana da Amazônia - FAMAZ

Av. Visc. de Souza Franco, 72 , Reduto,

66053-00, Belém - PA, Brasil

Tel.: (91) 99800-0505

E-mail: thiagoceleira@gmail.com

\section{Informações sobre os autores}

TCS é acadêmico do curso de Medicina da Faculdade Metropolitana da Amazônia - FAMAZ.

UTFHA é acadêmica do curso de Medicina da Faculdade Metropolitana da Amazônia - FAMAZ.

DAOB é graduada em Medicina pela Universidade Federal do Pará (UFPA); gastroenterologista pela Universidade Federal de São Paulo (UNIFESP - EMP); mestra e doutoranda em Gastroenterologia pela Universidade Federal de São Paulo (UNIFESP - EMP) e médica do Hospital Ophir Loyola.

\section{Contribuição dos autores}

TCS e UTFHA realizaram a parte de busca de artigos assim como a redação do artigo. DAOB realizou a idealização do tema e correção dos textos.

Todos os autores leram e aprovaram a versão final submetida ao Pará Research Medical Journal. 